\title{
Low Dimensional Temporal Organization of Spontaneous Eye Blinks in Adults with Developmental Disabilities and Stereotyped Movement Disorder
}

\author{
Mei-Hua Lee, \\ The Pennsylvania State University \\ James W. Bodfish, \\ University of North Carolina, Chapel-Hill \\ Mark H. Lewis, and \\ University of Florida \\ Karl M. Newell \\ The Pennsylvania State University
}

\section{Abstract}

This study investigated the mean rate and time-dependent sequential organization of spontaneous eye blinks in adults with intellectual and developmental disability (IDD) and individuals from this group that were additionally categorized with stereotypic movement disorder (IDD+SMD). The mean blink rate was lower in the IDD+SMD group than the IDD group and both of these groups had a lower blink rate than a contrast group of healthy adults. In the IDD group the $\mathrm{n}$ to $\mathrm{n}+1$ sequential organization over time of the eye blink durations showed a stronger compensatory organization than the contrast group suggesting decreased complexity/dimensionality of eye-blink behavior. Very low blink rate (and thus insufficient time series data) precluded analysis of time-dependent sequential properties in the IDD+SMD group. These findings support the hypothesis that both IDD and SMD are associated with a reduction in the dimension and adaptability of movement behavior and that this may serve as a risk factor for the expression of abnormal movements.

\section{Keywords}

Stereotypic movement disorders; eye blinks, intellectual and developmental disabilities

\begin{abstract}
Stereotypic movement disorders (SMD) are particularly prevalent in individuals with intellectual and developmental disability (IDD) (Baumeister \& Forehand, 1973; Cooper \& Dourish, 1990; Sprague \& Newell, 1996), though stereotypy is also evident in a number of other population groups and species, including human infants (Thelen, 1996) and caged animals (Berkson, 1967; Mason \& Rushen, 2007). The pathophysiological basis of stereotypies in neurodevelopmental disorders is not well understood. There is growing evidence, however,
\end{abstract}

\section{(C) 2009 Elsevier Ltd. All rights reserved.}

Mailing Address: Mei-Hua Lee Department of Kinesiology 266 Recreation Building The Pennsylvania State University University Park, PA 16802 Tel: (814) 863-4037 Fax: (814)863-7360 mx158@psu.edu.

Publisher's Disclaimer: This is a PDF file of an unedited manuscript that has been accepted for publication. As a service to our customers we are providing this early version of the manuscript. The manuscript will undergo copyediting, typesetting, and review of the resulting proof before it is published in its final citable form. Please note that during the production process errors may be discovered which could affect the content, and all legal disclaimers that apply to the journal pertain. 
that these behaviors are associated with alterations in cortical-basal ganglia circuitry (Lewis, Tanimura, Lee, \& Bodfish, 2007). This is consistent with long-standing observations that dopamine agonists (e.g., amphetamine) acting on the basal ganglia reliably induce stereotyped behavior in a wide range of animal species (see Cooper \& Dourish, 1990). In addition, stereotyped behavior that has been shown to be a predictable consequence of early social deprivation in non-human primates was shown to be associated with dopamine receptor supersensitivity (Lewis, Gluck, Beauchamp, Keresztury, \& Mailman, 1990), loss of dopamine innervation in striatum and dopamine cells in substantia nigra, and decreases in striatal neuropeptide staining (Martin, Spicer, Lewis, Gluck, \& Cork, 1991).

It has been proposed that spontaneous blink rate is a valid and robust behavioral index of the status of dopaminergic function in the system (Bodfish, Powell, Golden, \& Lewis, 1995; Lewis, Gluck, Bodfish, Beauchamp, \& Mailman, 1996). The mean rate of spontaneous eye blinks has been shown to vary over changes of age through the life span and as a function of particular disease states (Caplan et al, 1996; Schellini, Sampaio, Hoyama, Cruz, \& Padovani, 2005; Zamerkin, Stevens, \& Pittman, 1979). Importantly, in the context of this paper, the mean rate of spontaneous eye blinks has been shown to be lower in adults diagnosed with Stereotyped Movement Disorder (SMD) (Bodfish et al., 1995; MacLean et al., 1985; Roebel \& MacLean, 2007).

The hypothesis of a relation of the mean duration between eye blinks and the dopaminergic status of the system provides a natural and relatively convenient experimental behavioral index of pathophysiology of SMD. However, the mean of cyclical processes does not capture or exploit the richness of the dynamics of a time series and may even be misleading as to the dynamics and clinical status of a system (Bassingthwaite, Liebovitch, \& West, 1994; West, 2006). For example, there may be no difference between the mean heart rate of individuals over a segment of time but the heart rate could differ significantly in the time- and frequencydependent structure (Lipsitz \& Goldberger, 1992). Thus, an examination of the dynamics of a time series of spontaneous eye blinks beyond the mean rate could in principle provide a more informative and robust evaluation of SMD and related abnormal movements in IDD.

A striking example of this time series approach in repetitive movement disorders is that of Peterson and Leckman (1998) who studied the time dependent relations of the time intervals between tics through a variety of analysis methods. The results showed that the tic intervals were not statistically independent and that the frequency distribution of the tic interval duration followed an inverse power law 1 . These findings together with those from other analyses gave preliminary evidence for the presence of a fractal, deterministic and possibly chaotic processes in the tic time series. Furthermore, this study showed that time series methods are useful in the analysis of repetitive movement disorders and the characterization of symptom severity.

In a series of previous studies investigating the dynamics of stereotyped movements in posture and action tasks we have shown that the sequential properties of the movement time series reveal important properties of the system dynamics and differences between population groups with IDD (see Newell, 1996, 1997). A general finding is that the dimension of the movement output is progressively lower in instrumental tasks in individuals who display SMD + IDD when contrasted with individuals with IDD who do not exhibit SMD (Bodfish, Parker, Lewis, Sprague, \& Newell, 2001). Expressed another way, SMD progressively reduces the number of functional degrees of freedom (dimension) of the motor behavior beyond that of individuals with IDD but not recognized as exhibiting SMD, even in goal-oriented movement tasks.

\footnotetext{
${ }^{1}$ In an inverse power law the distribution of the data set is inversely proportional to the frequency of the form $1 / f^{i}$ where $f$ is a frequency and $i$ is a positive exponent called the spectral coefficient. When the data are presented in a log-log format the data appear as a straight line over a certain range. See West (2006).
} 
In this study we examined the organization of the spontaneous eye blinks of individuals with IDD and of age-matched healthy adults, a contrast that has not been made directly. In the IDD group we also contrasted the organization of eye blinks of individuals categorized with SMD and those that were not. Based on the previous findings of the progressive reduced dimension of motor behavior in IDD and IDD+SMD (Newell, 1996, 1997) it was hypothesized that the organization of eye blinks would also show reduced dimensionality with IDD that would be further reduced with SMD. Thus, we anticipated that there would be a reduction in both the mean blink rate and the dimension of the dynamics of natural eye blinks in intellectual and developmental disability (IDD) that would be magnified in individuals also diagnosed with SMD.

\section{Methods

The participant pool was composed of 2 groups: one with Intellectual and Developmental Disability (IDD) and a contrast group of aged matched healthy adults. The IDD group was further sub-divided into two subgroups: participants with comorbid Stereotyped Movement Disorder (IDD+SMD) and the remaining participants who did not display stereotyped behaviors (IDD). The participants in the IDD group resided at a public residential facility and were recruited from a larger group of persons with IDD who resided at the facility $(\mathrm{N}=290)$.

The recruitment was based on the following criteria: (a) adult between the ages of 18 and 60 years, (b) diagnosed with severe IDD, (c) ambulatory, (d) free of chronic medical conditions including seizure disorders, (e) not receiving psychotropic medication, and (f) parent or guardian provided informed consent for participation in this study. The IDD only subgroup had 25 participants, 16 males and 9 females (mean (SD) age $=48.68$ (9.58) years). The IDD + SMD subgroup included 8 participants, 6 males and 2 females (mean (SD) age $=46.1$ (9.71) years), and it was defined as those individuals from the IDD group, who: (a) received a minimum score of 2 on the Stereotype (STY) Checklist (used to identify presence of stereotyped movements during a standard motor exam, Bodfish et al., 1995; and (b) received a minimum score of 8 on the Behavior Problems Inventory (BPI) Stereotype Subscale (used to determine minimum cut-off for frequency/severity of stereotyped movements, Rojahn, Matson, Lott, Esbensen, \& Smalls, 2001). Diagnosis of IDD in both the IDD and the IDD + SMD groups was determined based on chart review (recent cognitive and adaptive behavior assessments) and using DSM-IV criteria for Mental Retardation.

The contrast group included 19 participants, 9 males and 10 females (mean (SD) age $=44.3$ (14.27) years), who were adults (between ages of 18 and 60 years), healthy (no chronic medical conditions), ambulatory, free of any neurological, endocrine (hormone) or neuromotor (nerve/ movement) disorders, and who were not receiving psychotropic medication. The contrast sample was recruited from a university setting. The protocol was approved by both The Pennsylvania State University IRB and the Riddle Institute IRB.

Of the 52 participants, 24 individuals met the specific inclusion criteria for the time series analyses of the sequential structure of eye blink duration that was an average eye blink rate of more than 10 blinks/min (this rate was required to provide a robust amount of data for the sequential analysis). Of these 24 selected participants, 13 were males and 11 were females. These selected participants were divided by group as follows: 7 males and 2 females were in the IDD group, 1 male were in the SIDD+SMD group, and 5 males and 9 females were in the contrast group. Table 1 shows a summary of demographic information for the entire participant pool and the subset selected for further time series analyses. 


\section{Procedures}

Videotaped records of natural, spontaneous eye-blink behavior were obtained during the course of a standard motor exam. A 3-5 min period of quiet-sitting/ no interaction was inserted in the exam as a probe for spontaneous eye-blinks. Participants were seated in a chair against the wall in a clinical exam room and were asked to look towards the camera for $3 \mathrm{~min}$ in the observation room. The examination room was free of external noise and interruption. Spontaneous eye blink was defined as the "simultaneous closure of the upper and lower eyelids of both eyes" (MacLean et al., 1985). The video recordings were coded by two observers using the Noldus Observer 5.0 program. The inter-observer reliability was determined for $25 \%$ of all observation sessions (across all groups). The average inter-observer agreement was $93.2 \%$.

\section{Results \\ Mean Eye blink rate}

Figure 1 shows the mean and between-participant standard deviation of blink rate for each group. A one way ANOVA showed significant group differences in the mean eye blink rate, $F(2,49)=10.41, p<0.05$. Post-hoc analyses revealed significant differences in mean eye blink rate between all groups; for IDD (mean $=8.91$ blinks $/ \mathrm{min}$ ) versus IDD+SMD (mean $=4.42$ blinks $/ \mathrm{min}$ ); for IDD+SMD versus Contrast (mean $=19.55 \mathrm{blinks} / \mathrm{min}$ ); and for IDD versus Contrast. In summary, the mean rate of natural eye blinks was significantly lower in the IDD + SMD group compared to the IDD group, and both were lower than the contrast healthy group.

\section{Sequential Blink Interval Relations}

To identify the sequential structure in the blink-to-blink relations of the spontaneous eye movements the differences between adjacent inter-blink durations were calculated.

Differencing data takes out the slower larger amplitude trends in the time series and gives emphasis to the moment to moment fluctuations rather than the mean trends of the dominant time scale(s) in the data (Williams, 1997).

Figure 2 shows representative participant plots for both the IDD and contrast groups of blink duration differences for $\mathrm{n}$ to $\mathrm{n}+1$ blinks. Thus, the sequential spontaneous eye blink durations of a complete trial are plotted in a two dimensional space of $n$ to $n+1$ with each point representing trial 1 with trial 2 , trial 2 with trial 3 , trial 3 with trial 4 and so on. The very low blink rate for the IDD+SMD group provided insufficient data for a sequential analysis of this group. The figure shows that the trend for both the IDD and contrast groups is a compensatory relation between successive blinks with the IDD participants having a less adaptable sequential structure. That is, there was a trend for the adjacent blink interval durations to be shorter after a relatively long duration blink interval, and vice-versa.

An independent sample t-test revealed that the slope of the regression line for the $\mathrm{n}$ to $\mathrm{n}+1$ blink duration differences was significantly higher in the IDD $(M=-0.63)$ than contrast $(M=$ -0.47 ) groups, $t(21)=-2.21, p>.05$. Furthermore, an independent sample t-test showed that there was a significant difference in the square of a correlation coefficient $\left(\mathrm{R}^{2}\right)$ of the regression line between IDD $(M=-0.39)$ and control group $(M=-0.23), t(21)=2.85, p>.05$. Thus, the fit of the regression line to the $\mathrm{n}$ to $\mathrm{n}+1$ blink data was also stronger in the IDD than the contrast group. These differences in the sequential organization of the inter blink interval data showed that the IDD group had a stronger and more rigid pattern of compensation from blink to blink than the contrast group. Finally, there was a significant difference in the slope of the regression line for mean blink rate and slope of $\mathrm{n}$ to $\mathrm{n}+1$ fit for the IDD group ( $\mathrm{r}=.32)$ and the contrast group ( $\mathrm{r}=.14)$. 
There was no significant relation $(\mathrm{p}>.05)$ between the slope of the $\mathrm{n}$ to $\mathrm{n}+1$ regression line and the number of data points in the time series $\left(R^{2}=0.13\right.$ for IDD; $R^{2}=0.01$ Contrast). Furthermore, a significant compensatory relation was not present in either group when the analysis was conducted on $n$ to $n+2$ or $n$ to $n+3$ relations. This confirms that the compensatory behavior in natural eye blinks is predominantly an adjacent interval $n$ to $n+1$ blink phenomenon.

\section{Discussion}

The findings of this study showed systematic changes in the rate and also in the dimension and organization of the duration between spontaneous eye blinks as a function of IDD only and also for SMD in the context of IDD. The obtained mean blink rate of these groups provided the first evidence that blink rate is reduced in individuals with IDD and replicated previous findings that blink rate is reduced in IDD+SMD (Bodfish et al., 1995; MacLean et al.,1985). Our study also reveals, however, that the sequential organization of natural eye blinks is of a lower dimension in individuals with IDD in that it has a stronger compensatory and more rigid sequential structure in the $\mathrm{n}$ to $\mathrm{n}+1$ blink durations.

The robust group differences in the mean and between-participant variability of natural blink rate is consistent with the proposal that natural blink rate is a useful behavioral index of the status of dopaminergic function of the system (Bodfish et al., 1995; Lewis et al., 1996). Thus, spontaneous eye-blink rate may serve as a practical non-invasive marker of pathophysiology in SMD. It is noteworthy that the differences in blink rate between the groups are substantial in that the IDD group has about twice the rate of the IDD+SMD group, while the contrast group of healthy age-matched adults has about twice the blink rate of the IDD group. Indeed, the IDD +SMD group mean natural blink rate of 4.42 blinks/min is very low compared to most other population groups (Caplan et al., 1996; Schellinei et al., 2005; Zammerkin et al., 1979).

Previous studies of blink rate in SMD have only involved comparison to an IDD / no SMD contrast group (Roebel \& MacLean, 2007 Bodfish et al., 1995; MacLean et al., 1985). Thus, the status of IDD by itself and in comparison to typically development (non-IDD) with respect to average levels of blink rate was unknown. The inclusion of a non-IDD contrast group in the present study allowed us to examine the relation of lowered blink rate to IDD independent of SMD. The finding that blink rate was significantly reduced in IDD relative to the non-IDD contrast group, suggests that relative hypodopaminergic status (as estimated by blink rate) may exist as a general feature of severe IDD. If this is the case then this may help explain why IDD is a significant risk factor for the development of SMD (Lewis et al., 1996), given that hypodopaminergic status is associated with the development of stereotyped behavior in a variety of human and animal conditions.

The analysis of the sequential time-dependent structure of the natural eye blinks revealed in addition that the time-dependent organization of the pattern of eye blinks was different in IDD from the healthy contrast group. This trend was independent of the mean blink rate of each group as the exemplar individual $\mathrm{n}$ to $\mathrm{n}+1$ plots of blink duration in Figure 2 show. Overall, the analysis of the $n$ to $n+1$ relation of blink duration showed that the IDD group had a stronger and less adaptable compensatory blink structure than the healthy contrast group. This pattern of findings with eye blinks is consistent with the general proposition that IDD and SMD progressively reduce the dimension of movement behavior (Newell, 1996,1997), as already shown in standing posture, sitting, arm and finger tremor behavioral activities (e.g., Bodfish et al., 2001;Newell, Gao, \& Sprague, 1995;Sprague, Deutsch \& Newell, 2007).

A limitation of the study is that the low mean blink rate of the IDD+SMD group made it impossible to obtain a valid analysis of the sequential structure of the time series. To generate in this group a timeseries that had sufficient data points would require a considerably longer 
duration of data collection - a factor that also increases the likelihood of nonstationarity in the data. Nevertheless, it would be useful to extend the kind of analysis of spontaneous eye blinks conducted here to a longer time scale in all the population groups studied. The hypothesis that needs to be tested is whether a SMD group has a lower dimensional time-dependent structure to the organization of eye blinks than an IDD group. Longer time series with more data points would also provide the data necessary for a fractal analysis of the inter-blink interval durations (Peterson \& Leckman, 1998). Overall, however, the findings support the hypothesis that hypodopaminergic function is progressively reduced in IDD and individuals additionally diagnosed with SMD, and that loss of dopamine function leads to a reduction in the dimension and adaptability of eye movement behavior.

\section{Acknowledgments}

We would like to thank Tim Benner, Jamie Clary and Dawn Parker, Nancy Poteet and Vicki Harper for assistance with data collection for this study and for technical help. This study was supported in part by NIH HD 46918 .

\section{References}

Bassingthwaighte, JB.; Liebovitch, LS.; West, BJ. Fractal physiology. Oxford University Press; Oxford: 1994.

Baumeister, AA.; Forehand, R. Stereotyped acts. In: Ellis, NR., editor. International review of research in mental retardation. Vol. 6. Academic Press; New York: 1973. p. 55-96.

Berkson, G. Abnormal stereotyped motor acts. In: Zubin, J.; Hunt, HF., editors. Comparative psychopathology - animal and human. Grune \& Stratton; New York: 1967. p. 76-94.

Bodfish JW, Powell SB, Golden RN, Lewis MH. Blink rate as an index of dopamine function in adults with mental retardation and repetitive behavior disorders. American Journal on Mental Retardation 1995;99:335-344. [PubMed: 7695876]

Bodfish JW, Parker DE, Lewis MH, Sprague RL, Newell KM. Stereotypy and motor control: Differences in the postural stability dynamics of persons with stereotyped and dyskinetic movement disorders. American Journal on Mental Retardation 2001;106:123-134. [PubMed: 11321603]

Caplan R, Guthrie D, Komo S. Blink rate in children with attention-deficit-hyperactivity disorder. Biological Psychiatry 1996;39:1032-1038. [PubMed: 8780838]

Cooper, SJ.; Dourish, CT., editors. Neurobiology of stereotyped behavior. Clarendon Press; Oxford: 1990.

Lewis MH, Tanimura Y, Lee L, Bodfish JW. Animal models of restricted repetitive behavior in autism. Behavioural Brain Research 2007;176:66-74. [PubMed: 16997392]

Lewis MH, Gluck JP, Beauchamp AJ, Keresztury MF, Mailman RB. Long term effects of early social isolation in Macaca mulatta: In vivo evidence for changes in dopamine receptor function. Brain Research 1990;513:67-73. [PubMed: 1693540]

Lewis, MH.; Gluck, JP.; Bodfish, JW.; Beauchamp, AJ.; Mailman, RB. Neurobiological basis of stereotyped movement disorder. In: Sprague, RL.; Newell, KM., editors. Stereotypies: Brainbehavior relationships. American Psychological Association; Washington, D. C.: 1996. p. 37-68.

Lipsitz LA, Goldberger AL. Loss of 'complexity' and aging: Potential applications of fractals and chaos theory to senescence. Journal of the American Medical Association 1992;267:1806-1809. [PubMed: 1482430]

MacLean WE Jr. Lewis MH, Bryson-Brockman WA, Ellis DN, Arendt RE, Baumeister AA. Blink rate and stereotyped behavior: Evidence for dopamine involvement? Biological Psychiatry 1985;20:1321-1325. [PubMed: 4063422]

Martin LJ, Spicer DM, Lewis MH, Gluck JP, Cork LC. Social deprivation of infant rhesus monkeys alters the chemoarchitecture of the brain. Journal of Neuroscience 1991;11:3344-3358. [PubMed: 1682426]

Mason, G.; Rushen, J. Stereotypic animal behavior: Fundamentals and applications to welfare. Oxford University Press; Oxford: 2007. 
Newell, KM. The dynamics of stereotypic behaviors. In: Sprague, RL.; Newell, KM., editors. Stereotypies: Brain-behavior relationships. American Psychological Association; Washington, D. C.: 1996.

Newell, KM. Motor skills and mental retardation. In: MacLean, W., editor. Handbook of mental deficiency. Vol. 3. Erlbaum; Hillsdale, NJ: 1997. p. 275-308.

Newell KM, Gao F, Sprague RL. The dynamics of finger tremor in tardive dyskinesia. Chaos 1995;5:4347. [PubMed: 12780153]

Peterson BS, Leckman JF. The temporal dynamics of tics in Gilles de la Tourette syndrome. Biological Psychiatry 1998;44:1337-1348. [PubMed: 9861477]

Roebel AM, MacLean WE Jr. Spontaneous eye-blinking and stereotyped behavior in older persons with mental retardation. Research in Developmental Disabilities 2007;28:37-42. [PubMed: 16359842]

Rojahn J, Matson JL, Lott S, Esbensen AJ, Small Y. The Behavior Problems Inventory: an instrument for the assessment of self-injury, stereotyped behavior, and aggression/destruction in individuals with developmental disabilities. Journal of Autism and Developmental Disorders 2001;31:577-588. [PubMed: 11814269]

Schellini SA, Sampaio AA, Hoyama E, Cruz AAV, Padovani CR. Spontaneous eye blink analysis in the normal individual. Orbit 2005;24:239-242. [PubMed: 16354632]

Sprague, RL.; Newell, KM., editors. Stereotypies: Brain-behavior relationships. American Psychological Association; Washington, D.C: 1996.

Sprague RL, Deutsch KM, Newell KM. Tremor frequency profile as a function of level of developmental disability. American Journal on Mental Retardation 2007;112:300-307. [PubMed: 17559296]

Thelen, E. Normal infant stereotypies: A dynamic systems approach. In: Sprague, RL.; Newell, KM., editors. Stereotypies: Brain-behavior relationships. American Psychological Association; Washington, D. C.: 1996. p. 139-166.

West, BJ. Where medicine went wrong: Rediscovering the path to complexity. World Scientific; New Jersey: 2006.

Williams, GP. Chaos theory tamed. Joseph Henry Press; Washington, D. C.: 1997.

Zametkin AJ, Stevens JR, Pittman R. Ontogeny of spontaneous blinking and of habituation of the blink reflex. Annals of Neurology 1979;5:453-457. [PubMed: 223495] 


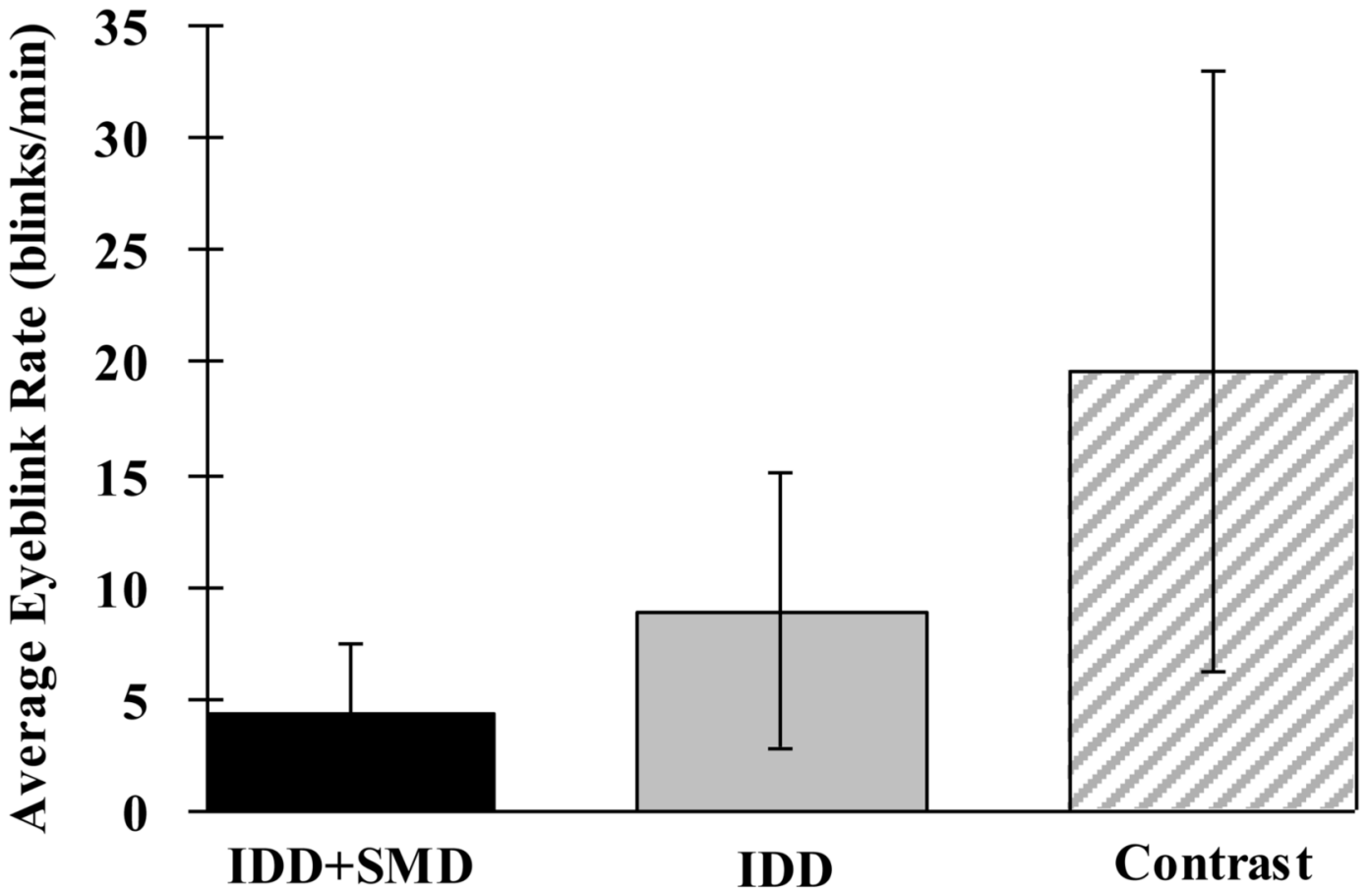

Figure 1.

Mean blink rate (and between-participant standard deviation) in the IDD+SMD, IDD and contrast groups. 

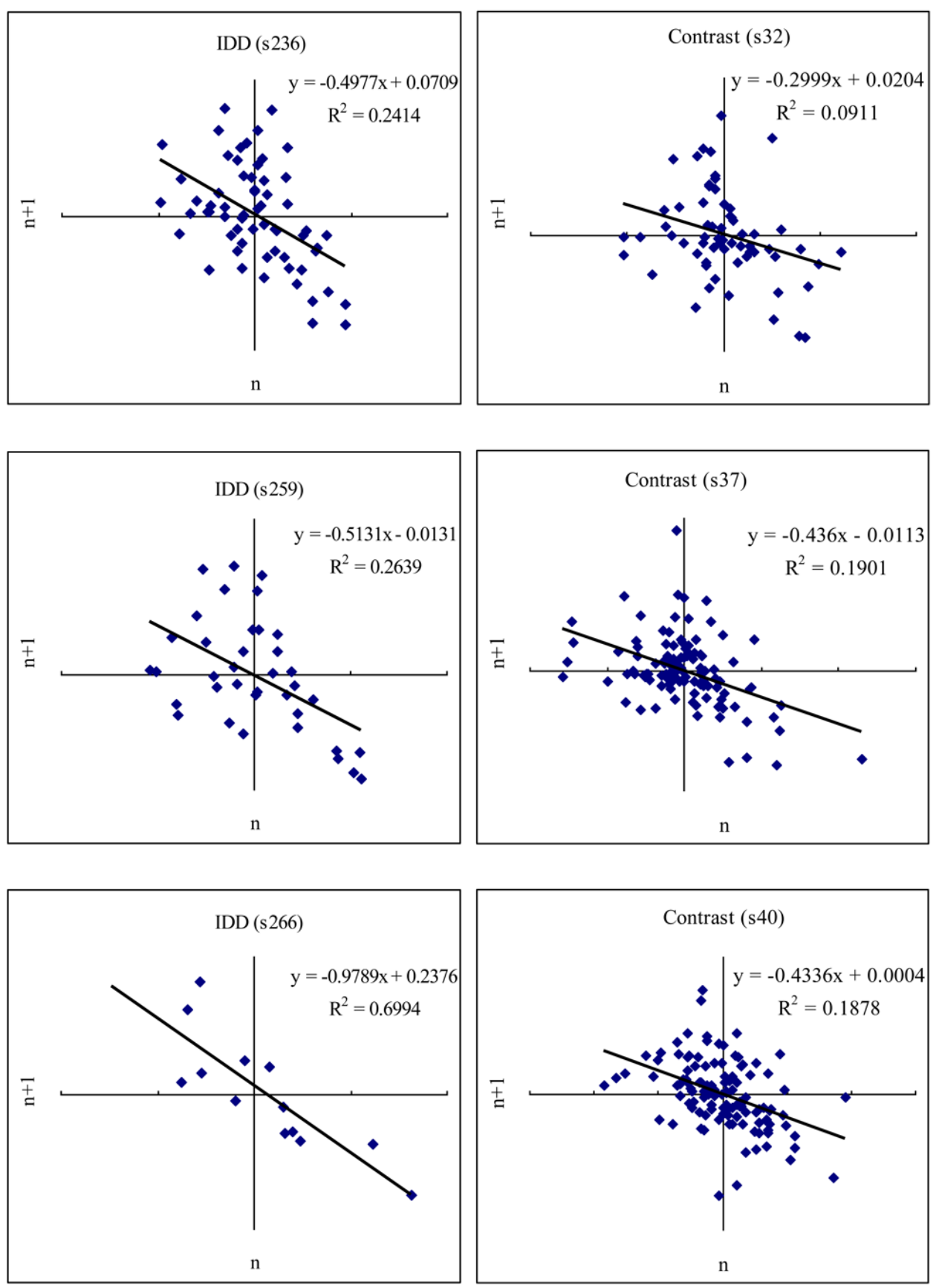

Figure 2.

Representative example scatterplots of the sequence relations of $n$ to $n+1$ blink interval data for 3 participants from each of the IDD and contrast groups. 


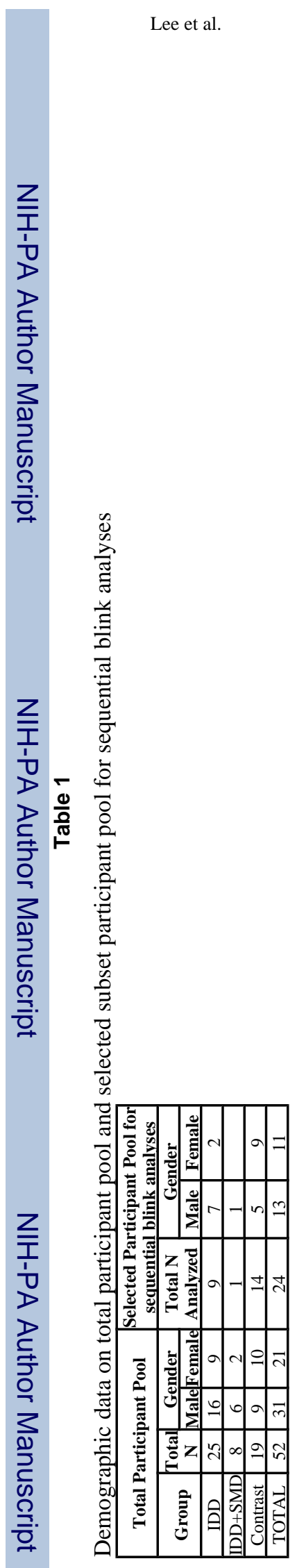

Page 10

Res Dev Disabil. Author manuscript; available in PMC 2011 January 1. 